\title{
DIAGONAL CAUCHY SPACES
}

\section{D.C. Kent and G.D. Richardson}

A diagonal condition is defined which internally characterises those Cauchy spaces which have topological completions. The $T_{2}$ diagonal Cauchy spaces allow both a finest and a coarsest $T_{2}$ diagonal completion. The former is a completion functor, while the latter preserves uniformisability and has an extension property relative to $\theta$-continuous maps.

\section{INTRODUCTION}

In 1954, Kowalsky [2] defined a diagonal axiom for convergence spaces subject to which every pretopological space is topological. In 1967, Cook and Fischer [1] gave a stronger version of the Kowalsky axiom relative to which a larger class of convergence spaces was shown to be topological. In [4], we showed that any convergence space satisfying the Cook-Fischer diagonal axiom is topological.

In this paper, we introduce a diagonal axiom for Cauchy spaces which reduces to the Cook-Fischer axiom when the Cauchy space is complete. A diagonal Cauchy space is one which satisfies this axiom. We show that a Cauchy space is diagonal if and only if every Cauchy equivalence class contains a smallest Cauchy filter, and this smallest Cauchy filter has a base of open sets. Equivalently, a Cauchy space is diagonal if and only if it allows a diagonal (that is, topological) completion. The category of diagonal Cauchy spaces and Cauchy continuous maps is shown to be a topological category.

It is shown that a $T_{2}$ diagonal Cauchy space has both a finest and a coarsest $T_{2}$ diagonal completion. The "fine diagonal completion" determines a completion functor on the category of $T_{2}$, diagonal Cauchy spaces. The "coarse diagonal completion" is finer than any $T_{3}$ completion, and thus if a $T_{3}$, diagonal completion exists, it necessarily coincides with the coarse diagonal completion. In particular, the coarse diagonal completion preserves uniformisability. A condition is given which is necessary and sufficient for the coarse diagonal completion of a $T_{3}$, diagonal Cauchy space to be $T_{3}$, and this is followed by an example which shows that the coarse diagonal completion of a totally bounded, $T_{3}$, diagonal Cauchy space can fail to be either $T_{3}$ or totally bounded.

Finally, we show that the coarse diagonal completion, although not functorial, has the following interesting extension property: Any Cauchy continuous map between $T_{2}$, diagonal Cauchy spaces has a $\theta$-continuous extension to the respective coarse diagonal completions.

Received 30th October, 1995

Copyright Clearance Centre, Inc. Serial-fee code: 0004-9729/96 \$A2.00+0.00. 


\section{Diagonal Cauchy spaces}

Let $X$ be a set, $F(X)$ the set of all (proper) filters on $X$, and $2^{X}$ the set of all subsets of $X$. For $x \in X$, let $\dot{x}$ be the fixed ultrafilter generated by $\{x\}$. For $\mathcal{F}, \mathcal{G} \in \mathbf{F}(X)$, we write $\mathcal{F} \leqslant \mathcal{G}$ if $\mathcal{F} \subseteq \mathcal{G}$. If $F \cap G \neq \emptyset$, for all $F \in \mathcal{F}$ and $G \in \mathcal{G}$, let $\mathcal{F} \vee \mathcal{G}$ denote the filter generated by $\{F \cap G: F \in \mathcal{F}, G \in \mathcal{G}\}$. If, on the other hand, there are $F \in \mathcal{F}$ and $G \in \mathcal{G}$ such that $F \cap G=\emptyset$, we say that " $\mathcal{F} \vee \mathcal{G}$ fails to exist".

Definition 1.1: A convergence structure $q$ on a set $X$ is a function $q: \mathbf{F}(X) \rightarrow$ $2^{X}$ such that:

(a) $x \in q(\dot{x})$, for all $x \in X$;

(b) $\mathcal{F} \leqslant \mathcal{G} \Rightarrow q(\mathcal{F}) \subseteq q(\mathcal{G})$;

(c) $x \in q(\mathcal{F}) \Rightarrow x \in q(\mathcal{F} \cap \dot{x})$.

The statement $x \in q(\mathcal{F})$ means " $\mathcal{F} q$-converges to $x$," which is also written " $\mathcal{F} \stackrel{q}{\rightarrow} x$." If $p, q$ are convergence structures on $X, p \leqslant q$ if and only if $\mathcal{F} \stackrel{q}{\rightarrow} x$ implies $\mathcal{F} \stackrel{p}{\rightarrow} x$. If $p \leqslant q$, we say that " $p$ is coarser than $q$ " or " $q$ is finer than $p$." If $q$ is a convergence structure on $X$, then $(X, q)$ is a convergence space.

Given a convergence space $(X, q)$ and $x \in X$, let $\mathcal{V}_{q}(x)$ denote the intersection of all filters which $q$-converge to $x$, and for a subset $A$ of $X$, let $I_{q}(A)=\{x \in A: A \in$ $\left.\mathcal{V}_{q}(x)\right\}$ and $c l_{q}(A)=\{x \in X:$ there is $\mathcal{F} \stackrel{q}{\rightarrow} x$ such that $A \in \mathcal{F}\} . \mathcal{V}_{q}(x)$ is called the $q$-neighbourhood filter at $x, I_{q}(A)$ is the q-interior of $A$, and $c l_{q}(A)$ is the $q$-closure of $A$. A subset $U$ of $X$ is defined to be $q$-open if $I_{q}(U)=U$. A convergence structure $q$ on $X$ is called a pretopology if $\mathcal{V}_{q}(x) \stackrel{q}{\rightarrow} x$, for each $x \in X$; a pretopology in which every neighbourhood filter has a base of $q$-open sets is a topology. For any convergence structure $q$ on $X$, the set of all $q$-open subsets of $X$ determines a topology $\tau_{q}$ on $X$, called the topological modification of $q$, which is the finest topology coarser than $q$.

In 1967, Cook and Fischer [1] introduced a "diagonal condition" for convergence spaces which we shall call Condition F. This condition is defined by means of a "compression operator" for filters which is defined as follows: If $J$ is any set, $\mathcal{F} \in \mathbf{F}(J)$, and $\sigma: J \rightarrow \mathbf{F}(X)$ is any function, let $\kappa \sigma \mathcal{F}=\bigcup_{F \in \mathcal{F}}(\bigcap \sigma(y): y \in F)$. Condition $F$ may now be stated for a convergence space $(X, q)$ as follows:

F: Let $J$ be any set, $\psi: J \rightarrow X$, and let $\sigma: J \rightarrow \mathbf{F}(X)$ have the property that $\psi(y) \geqslant \sigma(y)$ and $\sigma(y) \stackrel{q}{\rightarrow} \psi(y)$, for all $y \in J$. If $\mathcal{F} \in \mathbf{F}(J)$ is such that $\psi(\mathcal{F}) \stackrel{q}{\rightarrow} x$, then $\kappa \sigma \mathcal{F} \stackrel{q}{\rightarrow} x$.

In [4], the authors proved the following theorem.

THEOREM 1.2. A convergence structure $q$ on a set $X$ is a topology if and only if $(X, q)$ satisfies Condition $F$.

We shall proceed to formulate an analogous diagonal condition for Cauchy spaces. 
First we must review the basic theory of Cauchy spaces.

Definition 1.3: A Cauchy structure $\mathcal{C}$ on a set $X$ is a collection of filters on $X$ satisfying:

$\left(C_{1}\right) \quad \dot{x} \in \mathcal{C}$, for all $x \in X$;

$\left(C_{2}\right) \quad \mathcal{F} \in \mathcal{C}$ and $\mathcal{F} \leqslant \mathcal{G}$ implies $\mathcal{G} \in \mathcal{C}$;

$\left(C_{3}\right) \quad$ If $\mathcal{F}, \mathcal{G} \in \mathcal{C}$ and $\mathcal{F} \vee \mathcal{G}$ exists, then $\mathcal{F} \cap \mathcal{G} \in \mathcal{C}$.

If $\mathcal{C}_{1}, \mathcal{C}_{2}$ are Cauchy structures on $X$, we write $\mathcal{C}_{1} \leqslant \mathcal{C}_{2}$ if and only if $\mathcal{C}_{2} \subseteq \mathcal{C}_{1}$; in this case we say " $\mathcal{C}_{2}$ is finer than $\mathcal{C}_{1}$ " or " $\mathcal{C}_{1}$ is coarser than $\mathcal{C}_{2}$." A pair $(X, \mathcal{C})$ consisting of a set $X$ and a Cauchy structure $\mathcal{C}$ on $X$ is called a Cauchy space.

For each Cauchy space $(X, \mathcal{C})$, there is an associated convergence structure $q_{\mathcal{C}}$ on $X$ defined by $\mathcal{F} \stackrel{q_{\mathcal{C}}}{\rightarrow} x$ if and only if $\mathcal{F} \cap \dot{\boldsymbol{x}} \in \mathcal{C}$. A Cauchy space $(X, \mathcal{C})$ is $\mathbf{T}_{\mathbf{2}}$ (or Hausdorff) if $\dot{x} \cap \dot{y} \in \mathcal{C}$ implies $x=y$. Equivalently, $(X, \mathcal{C})$ is $T_{2}$ if and only if $\left(X, q_{\mathcal{C}}\right)$ is $T_{2}$ in the sense that each $q_{\mathcal{C}}$-convergent filter has a unique limit. A $T_{2}$ Cauchy space $(X, \mathcal{C})$ is $\mathbf{T}_{3}$ if $c l_{q_{\mathcal{C}}} \mathcal{F} \in \mathcal{C}$ whenever $\mathcal{F} \in \mathcal{C}$, complete if every filter $\mathcal{F}$ in $\mathcal{C}$ is $q_{\mathcal{C}}$-convergent, and totally bounded if every ultrafilter on $X$ is in $\mathcal{C}$.

Let $(X, \mathcal{C})$ be a Cauchy space. An equivalence relation $\sim$ on $\mathcal{C}$ is defined as follows: For $\mathcal{F}, \mathcal{G} \in \mathcal{C}, \mathcal{F} \sim \mathcal{G}$ if and only if $\mathcal{F} \cap \mathcal{G} \in \mathcal{C}$. If $\mathcal{F} \in \mathcal{C}$, let $[\mathcal{F}]_{\mathcal{C}}=\{\mathcal{G} \in \mathcal{C}: \mathcal{F} \sim \mathcal{G}\}$ be the equivalence class determined by $\mathcal{F}$; this equivalence class is denoted simply by $[\mathcal{F}]$ if there is no ambiguity.

If $(X, \mathcal{C})$ and $(Y, \mathcal{D})$ are two Cauchy spaces, $f:(X, \mathcal{C}) \rightarrow(Y, \mathcal{D})$ is Cauchy continuous if $\mathcal{F} \in \mathcal{C}$ implies $f(\mathcal{F}) \in \mathcal{D}$. If $f:(X, \mathcal{C}) \rightarrow(Y, \mathcal{D})$ is Cauchy continuous, then obviously $f:\left(X, q_{\mathcal{C}}\right) \rightarrow\left(Y, q_{\mathcal{D}}\right)$ is continuous in the sense that $\mathcal{F} \stackrel{q_{\mathcal{C}}}{\rightarrow} x$ implies $f(\mathcal{F}) \stackrel{q_{\mathcal{D}}}{\rightarrow} f(x)$. If $f:(X, \mathcal{C}) \rightarrow(Y, \mathcal{D})$ is a bijection such that $f$ and $f^{-1}$ are both Cauchy continuous, then $f$ is a Cauchy isomorphism.

Let $(X, \mathcal{C})$ be a Cauchy space and $A \subseteq X . \mathcal{F} \in \mathbf{F}(X)$ has a trace on $A$ if $F \cap A \neq \emptyset$, for all $F \in \mathcal{F}$; in this case $\mathcal{F}_{A}=\{F \cap A: F \in \mathcal{F}\}$ denotes the trace of $\mathcal{F}$ on $A . \quad \mathcal{C}_{A}=\left\{\mathcal{F}_{A}: \mathcal{F} \in \mathcal{C}, \mathcal{F}\right.$ has a trace on $\left.A\right\}$ is a Cauchy structure on $A$, and $\left(A, \mathcal{C}_{A}\right)$ is a Cauchy subspace of $(X, \mathcal{C})$. If $f:(X, \mathcal{C}) \rightarrow(Y, \mathcal{D})$ is such that $f:(X, \mathcal{C}) \rightarrow\left(f(X), \mathcal{D}_{f(X)}\right)$ is a Cauchy isomorphism, then $f:(X, \mathcal{C}) \rightarrow(Y, \mathcal{D})$ is called a Cauchy embedding.

We next define the diagonal Condition $\mathrm{D}$ for a Cauchy space $(X, \mathcal{C})$. The following notation will be useful: $\mathcal{N}_{\mathcal{C}}=\left\{\mathcal{G} \in \mathcal{C}: \mathcal{G}\right.$ is non- $q_{\mathcal{c}}$-convergent $\}$.

D: Let $J$ be any set, $\psi: J \rightarrow X \cup\left\{[\mathcal{G}]: \mathcal{G} \in \mathcal{N}_{\mathcal{C}}\right\}$, and let $\sigma: J \rightarrow \mathcal{C}$ be such that $\psi \dot{(y)} \geqslant \sigma(y)$ and $\sigma(y) \stackrel{q_{\mathcal{C}}}{\rightarrow} \psi(y)$ if $\psi(y) \in X$ and $\sigma(y) \in[\mathcal{G}]$ if $\psi(y)=[\mathcal{G}]$, where $\mathcal{G} \in \mathcal{N}_{\mathcal{C}}$. If $\mathcal{F}$ is a filter on $J$ such that $\psi(\mathcal{F})$ is finer than the filter on $X \cup\left\{[\mathcal{G}]: \mathcal{G} \in \mathcal{N}_{\mathcal{C}}\right\}$ generated by $\mathcal{H} \cap[\dot{\mathcal{H}}]$; for some $\mathcal{H} \in \mathcal{C}$, then $\kappa \sigma \mathcal{F} \in \mathcal{C}$. 
Observe that Condition $\mathrm{D}$ is equivalent to Condition $\mathrm{F}$ if $(X, \mathcal{C})$ is a complete Cauchy space (in which case $\mathcal{C}$ can be identified with $q_{\mathcal{C}}$ ).

A Cauchy space $(X, \mathcal{C})$ which satisfies Condition $\mathrm{D}$ will be called a diagonal Cauchy space. We shall now establish some properties of diagonal Cauchy spaces.

THEOREM 1.4. If $(X, \mathcal{C})$ is a diagonal Cauchy space, then $q_{c}$ is a topology.

Proof: By Theorem 1.2, it is sufficient to show that $\left(X, q_{c}\right)$ satisfies Condition F. If $J, \psi$, and $\sigma$ are as specified in Condition $F$ and $\mathcal{F}$ is a filter on $J$ such that $\psi(\mathcal{F}) \stackrel{q_{\mathcal{C}}}{\rightarrow} x$ (which is equivalent to $\psi(\mathcal{F}) \cap \dot{x} \in \mathcal{C}$ ), then we are dealing with a special case of Condition D where $\psi(J) \subseteq X$, and we conclude by the latter condition that $\kappa \sigma \mathcal{F} \in \mathcal{C}$. But $\psi(\mathcal{F}) \geqslant \kappa \sigma \mathcal{F}$, and $(\psi(\mathcal{F}) \cap \dot{x}) \vee \kappa \sigma \mathcal{F}$ exists, so by Definition $1.3\left(C_{3}\right)$, $(\kappa \sigma \mathcal{F}) \cap \dot{x} \in \mathcal{C}$, or in other words, $\kappa \sigma \mathcal{F} \stackrel{q_{\mathcal{C}}}{\rightarrow} x$. Thus Condition $\mathrm{F}$ is satisfied.

Propos IT I ON 1.5. If $(X, \mathcal{C})$ is a diagonal Cauchy space and $\mathcal{G} \in \mathcal{C}$, then there is $\mathcal{H} \in \mathcal{C}$ such that $\mathcal{H} \leqslant \mathcal{G}$ and $\mathcal{H}$ has a filter base of $q_{\mathcal{c}}$-open sets.

Proof: Let $J=X$ and let $\mathcal{G} \in \mathcal{C}$. Let $\sigma(x)=\mathcal{V}_{q_{c}}(x)$, the $q_{\mathcal{c}}$-neighbourhood filter at $x$, for all $x \in X$. Let $\mathcal{H}=\kappa \sigma \mathcal{G}$. If $G \in \mathcal{G}$, then $\bigcap_{x \in G} \sigma(x)=\bigcap_{x \in G} \mathcal{V}_{q_{\mathcal{C}}}(x)$ is a filter with a base of $q_{\mathcal{C}}$-open sets by Theorem 1.4, and $\mathcal{H}=\kappa \sigma \mathcal{G}=\bigcup_{G \in \mathcal{C}}\left(\bigcap_{x \in G} \sigma(x)\right)$ is likewise a filter with a base of $q_{\mathcal{C}}$-open sets. Note that $\mathcal{H} \in \mathcal{C}$ by Condition $\mathrm{D}$, and $\mathcal{H} \leqslant \mathcal{G}$ is obvious from the construction of $\kappa \sigma \mathcal{G}$.

Theorem 1.6. If $(X, \mathcal{C})$ is a diagonal Cauchy space, then for each $\mathcal{G} \in \mathcal{C},[\mathcal{G}]$ contains a smallest filter $\mathcal{G}_{\min }$, and $\mathcal{G}_{\min }$ has a filter base of $q_{\mathcal{c}}$-open sets.

PRoof: If $\mathcal{G} \stackrel{q_{\mathcal{C}}}{\rightarrow} x$, then $\mathcal{G}_{\min }=\mathcal{V}_{q_{\mathcal{C}}}(x)$, and $\mathcal{V}_{q_{\mathcal{C}}}(x)$ has a filter base of $q_{\mathcal{C}}$-open sets by Theorem 1.4. Assume $\mathcal{G} \in \mathcal{N}_{\mathcal{C}}$ and let $\left\{\mathcal{G}_{\alpha}: \alpha \in A\right\}$ be a set of filters in $\mathcal{G}$ such that $\bigcap[\mathcal{G}]=\bigcap\left\{\mathcal{G}_{\alpha}: \alpha \in A\right\}$. Let $J=X \cup A$. Let $\psi: J \rightarrow X \cup\left\{[\mathcal{H}]: \mathcal{H} \in \mathcal{N}_{\mathcal{C}}\right\}$ be defined by $\psi(x)=x$, for all $x \in X$, and $\psi(\alpha)=[\mathcal{G}]$, for all $\alpha \in A$. Let $\sigma: J \rightarrow \mathcal{C}$ be defined by $\sigma(x)=\mathcal{V}_{q_{C}}(x)$, for all $x \in X$ and $\sigma(\alpha)=\mathcal{G}_{\alpha}$, for all $\alpha \in A$. Let $\mathcal{F}$ be the filter on $J$ with filter base $\{G \cup A: G \in \mathcal{G}\}$. Then $\psi(\mathcal{F})$ is the filter generated by $\{G \cup\{[\mathcal{G}]\}: G \in \mathcal{G}\}$, and so by Condition $\mathrm{D}, \kappa \sigma \mathcal{F} \in \mathcal{C}$. Note that for each $G \cup A \in \mathcal{F}, \bigcap_{y \in G \cup A} \sigma(y) \leqslant \bigcap\left\{\mathcal{G}_{\alpha}: \alpha \in A\right\}=\bigcap[\mathcal{G}]$, and since $\kappa \sigma \mathcal{F} \leqslant \bigcap[\mathcal{G}]$ and $\kappa \sigma \mathcal{F} \in[\mathcal{G}], \kappa \sigma \mathcal{F}=\mathcal{G}_{\min }$ is the smallest filter in $[\mathcal{G}]$. The fact that $\mathcal{G}_{\min }$ has a base of $q_{\mathcal{c}}$-open sets follows by Proposition 1.5 .

Let DCHY be the category whose objects are diagonal Cauchy spaces and whose morphisms are Cauchy continuous maps.

THEOREM 1.7. DCHY is a topological category.

Proof: It suffices to show that Condition $D$ is preserved under the formation 
of initial structures. Let $\left\{\left(X_{\alpha}, \mathcal{C}_{\alpha}\right): \alpha \in A\right\}$ be a collection of Cauchy spaces, each satisfying Condition D. Let $X$ be a set and $f_{\alpha}: X \rightarrow X_{\alpha}$ a family of maps indexed by $A$. Let $\mathcal{C}$ be the initial Cauchy structure on $X$ induced by the collections $\left\{f_{\alpha}: \alpha \in A\right\}$ and $\left\{\left(X_{\alpha}, \mathcal{C}_{\alpha}\right): \alpha \in A\right\}$. As is well known, $\mathcal{F} \in \mathcal{C}$ if and only if $f_{\alpha}(\mathcal{F}) \in \mathcal{C}_{\alpha}$, for all $\alpha \in A$.

Let $J$ be any set, $\psi: J \rightarrow X \cup\left\{[\mathcal{G}]_{\mathcal{C}}: \mathcal{G} \in \mathcal{N}_{\mathcal{C}}\right\}$ and $\sigma: J \rightarrow \mathcal{C}$ such that $\psi(y) \geqslant \sigma(y)$ and $\sigma(y) \stackrel{q_{\mathcal{C}}}{\rightarrow} \psi(y)$ if $\psi(y) \in X$ and $\sigma(y) \in[\mathcal{G}]$ if $\psi(y)=[\mathcal{G}]$. Let $\mathcal{F} \in \mathbf{F}(J)$ be such that $\psi(\mathcal{F})$ is finer than the filter on $X \cup\left\{[\mathcal{G}]_{\mathcal{C}}: \mathcal{G} \in \mathcal{N}_{\mathcal{C}}\right\}$ generated by $\mathcal{H} \cap[\dot{\mathcal{H}}]$, for some $\mathcal{H} \in \mathcal{C}$. We must show $\kappa \sigma \mathcal{F} \in \mathcal{C}$.

Let $\alpha \in A$ be fixed. Define $\psi_{\alpha}: J \rightarrow X_{\alpha} \cup\left\{[\mathcal{G}]_{\mathcal{C}_{\alpha}}: \mathcal{G} \in \mathcal{N}_{\mathcal{C}_{\alpha}}\right\}$ as follows:

1. $\psi_{\alpha}(y)=f_{\alpha}(\psi(y))$ if $\psi(y) \in X$.

2. $\quad \psi_{\alpha}(y)=\lim f_{\alpha}(\mathcal{G})$ if $\psi(y)=[\mathcal{G}]_{\mathcal{C}}$ and $f_{\alpha}(\mathcal{G})$ is $\boldsymbol{q}_{\mathcal{C}_{\alpha}}$-convergent.

3. $\quad \psi_{\alpha}(y)=\left[f_{\alpha}(\mathcal{G})\right]_{\mathcal{c}_{\alpha}}$ if $\psi(y)=[\mathcal{G}]_{\mathcal{c}}$ and $f_{\alpha}(\mathcal{G})$ is not $q_{\mathcal{c}_{\alpha}}$-convergent.

Let $\sigma_{\alpha}: J \rightarrow \mathcal{C}_{\alpha}$ be defined by: $\sigma_{\alpha}(y)=\mathcal{V}_{q_{\mathcal{C}_{\alpha}}}\left(\psi_{\alpha}(y)\right)$ if $\psi_{\alpha}(y)$ is defined by 1 or $2 ; \sigma_{\alpha}(y)=f_{\alpha}\left(\mathcal{G}_{\min }\right)$ if $\psi_{\alpha}(y)$ is defined as in 3 .

Note that for all $y$ in $J, f_{\alpha}(\sigma(y)) \geqslant \sigma_{\alpha}(y)$. Let $\mathcal{F} \in \mathrm{F}(J)$ be as above. Then for each $\alpha \in A, \psi_{\alpha}(\mathcal{F})$ is finer than the filter on $X_{\alpha} \cup\left\{[\mathcal{G}]_{\mathcal{C}_{\alpha}}: \mathcal{G} \in \mathcal{N}_{\mathcal{C}_{\alpha}}\right\}$ generated by $f_{\alpha}(\mathcal{H}) \cap f_{\alpha}(\mathcal{H})$. By the assumption that $\left(X_{\alpha}, \mathcal{C}_{\alpha}\right)$ satisfies $\mathrm{D}$, we have that $\kappa \sigma_{\alpha}(\mathcal{F}) \in \mathcal{C}_{\alpha}$. But since $f_{\alpha}(\sigma(y)) \geqslant \sigma_{\alpha}(y)$ holds for all $y \in J$, it follows that $f_{\alpha}(\kappa \sigma \mathcal{F}) \geqslant \kappa \sigma_{\alpha} \mathcal{F}$, which implies $f_{\boldsymbol{\alpha}}(\kappa \sigma \mathcal{F}) \in \mathcal{C}_{\alpha}$. Since $\alpha \in A$ is arbitrary, $\kappa \sigma \mathcal{F} \in \mathcal{C}$.

\section{Diagonal Cauchy completions}

Let $(X, \mathcal{C})$ and $(Y, \mathcal{D})$ be Cauchy spaces and $\phi: X \rightarrow Y$. Then $((Y, \mathcal{D}), \phi)$ is a completion of $(X, \mathcal{C})$ if:

(1) $(Y, \mathcal{D})$ is complete;

(2) $\phi:(X, \mathcal{C}) \rightarrow(Y, \mathcal{D})$ is a dense embedding.

Here, "dense" means that $c l_{q_{\mathcal{D}}} \phi(X)=Y$, where $c l_{q_{\mathcal{D}}}$ denotes the $q_{\mathcal{D}}$-closure operator.

A completion $((Y, \mathcal{D}), \phi)$ of $(X, \mathcal{C})$ is said to be diagonal (respectively, $\left.\mathrm{T}_{2}\right)$ if $(Y, \mathcal{D})$ is a diagonal (respectively, $T_{2}$ ) Cauchy space. Since the properties of being diagonal and $T_{2}$ are both hereditary (in the former case, by Theorem 1.7), any Cauchy space having a diagonal (or $T_{2}$ ) completion must itself be diagonal (or $T_{2}$ ). Note that a complete Cauchy space $(Y, \mathcal{D})$ is diagonal if and only if any one of the following equivalent conditions holds: $(1)(Y, \mathcal{D})$ satisfies Condition $\mathrm{D} ;(2)\left(Y, q_{\mathcal{D}}\right)$ satisfies Condition $\mathrm{F}$; (3) $\left(Y, q_{D}\right)$ is a topological space.

TheOREM 2.1. The following statements about a Cauchy space $(X, \mathcal{C})$ are equivalent.

(1) $(X, \mathcal{C})$ is a diagonal Cauchy space. 
(2) Each equivalence class $[\mathcal{F}]$ in $\mathcal{C}$ contains a smallest filter $\mathcal{F}_{\min }$, and $\mathcal{F}_{\min }$ has a base of $q_{c}$-open sets.

(3) $(X, \mathcal{C})$ has a diagonal Cauchy completion.

Proof: $(1) \Rightarrow(2)$. This is Theorem 1.6.

$(3) \Rightarrow(1)$. This follows by a remark in the paragraph preceding the theorem.

$(2) \Rightarrow(3)$. Let $(X, \mathcal{C})$ be a Cauchy space as described in (2). If $\mathcal{F} \stackrel{q_{\mathcal{C}}}{\rightarrow} X$, then $\mathcal{F}_{\text {min }}=$ $\mathcal{V}_{q_{c}}(x)$, and it follows by (2) that $q_{c}$ is a topology.

Let $X^{\sim}=X \cup\left\{[\mathcal{F}]: \mathcal{F} \in \mathcal{N}_{\mathcal{C}}\right\}$, and let $\psi: X \rightarrow X^{\sim}$ be the identity injection. For each $A \subseteq X$, let $A^{\sim}=A \cup\left\{[\mathcal{F}]: \mathcal{F} \in \mathcal{N}_{\mathcal{C}}\right.$ and $\left.A \in \mathcal{F}_{\min }\right\}$. Noting that $(A \cap B)^{\sim}=$ $A^{\sim} \cap B^{\sim}$, let $\mathcal{G} \in \mathbf{F}(X)$, and define $\mathcal{G}^{\sim} \in \mathbf{F}\left(X^{\sim}\right)$ to be the filter with base $\left\{G^{\sim}\right.$ : $G \in \mathcal{G}\}$. Define $\mathcal{C}^{\sim}=\left\{\mathcal{A} \in \mathbf{F}\left(X^{\sim}\right): \exists \mathcal{F} \in \mathcal{C}\right.$ such that $\left.\mathcal{A} \geqslant \mathcal{F}_{\text {min }}^{\sim}\right\}$; it is a simple matter to verify that $\mathcal{C}^{\sim}$ is a Cauchy structure on $X^{\sim}$. For $\mathcal{F} \in \mathcal{C}, \psi(\mathcal{F}) \geqslant \mathcal{F}^{\sim}$ and $\psi^{-1}\left(\mathcal{F}^{\sim}\right)=\mathcal{F}$; from these observations, it follows that $\psi:(X, \mathcal{C}) \rightarrow\left(X^{\sim}, \mathcal{C}^{\sim}\right)$ is a Cauchy embedding. Since $[\dot{\mathcal{F}}] \geqslant \mathcal{F}_{\text {min }}^{\sim}$ for all $\mathcal{F} \in \mathcal{N}_{\mathcal{C}}, \mathcal{F}_{\text {min }}^{\sim} \cap[\dot{\mathcal{F}}] \in \mathcal{C}^{\sim}$, and hence $\mathcal{F}_{\text {min }}^{\sim} \stackrel{\boldsymbol{q}^{\sim}}{\rightarrow}[\mathcal{F}]$ for all $\mathcal{F} \in \mathcal{N}_{\mathcal{C}}$, where $q^{\sim}$ denotes the convergence structure associated with $\mathcal{C}^{\sim}$. Since $\mathcal{V}_{q_{\mathcal{C}}}(x)^{\sim} \stackrel{q^{\sim}}{\rightarrow} X$, for all $x \in X$, it follows that $\left(X^{\sim}, \mathcal{C}^{\sim}\right)$ is complete. Finally, $\mathcal{F}^{\sim} \stackrel{q^{\sim}}{\rightarrow}[\mathcal{F}]$ for all $\mathcal{F} \in \mathcal{N}_{\mathcal{C}}$ and $\psi(\mathcal{F}) \geqslant \mathcal{F}^{\sim}$ imply that $c l_{q} \sim \psi(X)=X^{\sim}$, so the embedding $\psi$ is dense.

It remains to show that $\left(X^{\sim}, \mathcal{C}^{\sim}\right)$ is a diagonal Cauchy space. First observe that $\left(X^{\sim}, q^{\sim}\right)$ is pretopological (meaning that the neighbourhood filter at each point in $X^{\sim}$ converges to that point). To show that each $q^{\sim}$-neighbourhood filter has a filter base of $q^{\sim}$-open sets, it suffices to show that $U^{\sim}$ is $q^{\sim}$-open whenever $U$ is $q_{c}$-open. If $x \in U^{\sim}$, then $U \in \mathcal{V}_{q_{\mathcal{C}}}(x)$, and hence $U^{\sim} \in \mathcal{V} q_{\mathcal{c}}(x)^{\sim}=\mathcal{V}_{q} \sim(x)$. If $[\mathcal{F}] \in U^{\sim}$, where $\mathcal{F} \in \mathcal{N}_{\mathcal{C}}$, then $U \in \mathcal{F}_{\min }$ implies $U^{\sim} \in \mathcal{F}_{\min }^{\sim}=\mathcal{V}_{q} \sim([\mathcal{F}])$. Thus $U^{\sim}$ is a $q^{\sim}$-open set, since it is a $q^{\sim-n e i g h b o u r h o o d ~ o f ~ e a c h ~ o f ~ i t s ~ p o i n t s, ~ a n d ~ t h e ~ p r o o f ~ i s ~ c o m p l e t e . ~}$

Observe that the implication $(2) \Rightarrow(1)$ in Theorem 2.1 is the converse of Theorem 1.6 .

Theorem 2.2. If $(X, \mathcal{C})$ is a $T_{2}$, diagonal Cauchy space, the diagonal completion $\left(\left(X^{\sim}, \mathcal{C}^{\sim}\right), \psi\right)$ constructed in the preceding proof is likewise $T_{2}$.

Proof: If $[\dot{\mathcal{F}}] \cap[\dot{\mathcal{G}}] \in \mathcal{C}^{\sim}$, where $\mathcal{F}, \mathcal{G} \in \mathcal{N}_{\mathcal{C}}$, then $\mathcal{F}_{\min } \cap \mathcal{G}_{\min } \in \mathcal{C}$, which implies $\mathcal{F}_{\min }=\mathcal{G}_{\min }$, and hence $[\mathcal{F}]=[\mathcal{G}]$. If $\dot{x} \cap \dot{y} \in \mathcal{C}^{\sim}$, then the intersection of the restrictions of $\dot{x}$ and $\dot{y}$ to $X$ is in $\mathcal{C}$, and since $(X, \mathcal{C})$ is $T_{2}, x=y$. If $[\dot{\mathcal{F}}] \cap \dot{x} \in \mathcal{C}^{\sim}$, where $\mathcal{F} \in \mathcal{N}_{\mathcal{C}}$ and $x \in X$, it follows easily that $\mathcal{F}_{\min } \stackrel{{ }^{q} \mathcal{C}}{\rightarrow} x$, a contradiction, and so this case is impossible. We conclude that $\left(X^{\sim}, \mathcal{C}^{\sim}\right)$ is $T_{2}$.

Let $(X, \mathcal{C})$ be any $T_{2}$ Cauchy space, let $X^{*}=\{[\mathcal{F}]: \mathcal{F} \in \mathcal{C}\}$, and let $\jmath: X \rightarrow X^{*}$ be defined by $\boldsymbol{\jmath}(\boldsymbol{x})=[\dot{\boldsymbol{x}}]$, for all $x \in X$. A $T_{2}$ completion $((Y, \mathcal{D}), \phi)$ of $(X, \mathcal{C})$ is said 
to be in standard form if $Y=X^{*}, \phi=\jmath$ and $\jmath(\mathcal{F}) \stackrel{q_{\mathcal{D}}}{\rightarrow}[\mathcal{F}]$. Reed, [3], showed that every $T_{2}$ completion of a Cauchy space $(X, \mathcal{C})$ is equivalent to one in standard form.

In case $(X, \mathcal{C})$ is a $T_{2}$, diagonal Cauchy space, it will be convenient to give a description of the preceding completion in standard form. Let $\mathcal{C}^{\circ}=\{\mathcal{F} \in \mathcal{C}: \mathcal{F}$ has a filter base of $q_{\mathcal{c}}$-open sets $\}$. Let $X^{*}$ and $\jmath$ be defined as in the preceding paragraph. For $A \subseteq X$ and $\mathcal{G} \in \mathcal{C}^{\circ}$, let $A^{*}=\left\{[\mathcal{F}] \in X^{*}: A \in \mathcal{F}_{\min }\right\}$ and let $\mathcal{G}^{*}$ be the filter on $X^{*}$ generated by $\left\{A^{*}: A \in \mathcal{G}\right\}$. Let $\mathcal{C}^{*}=\left\{\mathcal{A} \in \mathrm{F}\left(X^{*}\right)\right.$ : there is $\mathcal{F} \in \mathcal{C}^{\circ}$ such that $\left.\mathcal{F}^{*} \leqslant \mathcal{A}\right\}$. One easily verifies that $\left(\left(X^{*}, \mathcal{C}^{*}\right), \jmath\right)$ is a $T_{2}$, diagonal completion of $(X, \mathcal{C})$ in standard form, and if $\sigma: X^{\sim} \rightarrow X^{*}$ is defined by $\sigma(x)=[\dot{x}]$ for $x \in X$, and $\sigma([\mathcal{F}])=[\mathcal{F}]$ for $\mathcal{F} \in \mathcal{N}_{\mathcal{C}}$, then $\sigma:\left(X^{\sim}, \mathcal{C}^{\sim}\right) \rightarrow\left(X^{*}, \mathcal{C}^{*}\right)$ is a Cauchy isomorphism which makes the following diagram commute:

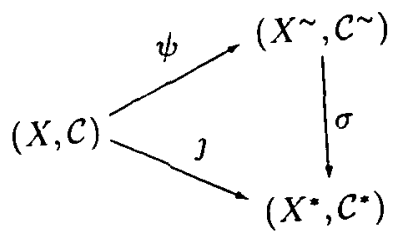

This establishes the equivalence of the completions $\left(\left(X^{*}, \mathcal{C}^{*}\right), \jmath\right)$ and $\left(\left(X^{\sim}, \mathcal{C}^{\sim}\right), \psi\right)$ of a $T_{2}$, diagonal Cauchy space $(X, \mathcal{C})$.

Theorem 2.3. Let $(X, \mathcal{C})$ be a $T_{2}$, diagonal Cauchy space. Let $\left(\left(X^{*}, \mathcal{C}^{*}\right), \jmath\right)$ be the $T_{2}$, diagonal completion of $(X, \mathcal{C})$ defined in the preceding paragraph, and let $\left(\left(X^{*}, \mathcal{D}\right), \jmath\right)$ be any $T_{2}$, diagonal completion of $(X, \mathcal{C})$ in standard form. Then $\mathcal{D} \subseteq \mathcal{C}^{*}$.

Proof: By analogy to the proof of Theorem 2.1, we see that sets of the form $U^{*}$, where $U$ is $q_{\mathcal{C}}$-open, form a basis for the topology $q^{*}$ of $\left(X^{*}, \mathcal{C}^{*}\right)$. Thus it suffices to show that if $U$ is $q_{\mathcal{C}}$-open, then $U^{*}$ is $q_{\mathcal{D}}$-open.

Let $U$ be $q_{\mathcal{c}}$-open and let $[\mathcal{F}] \in U^{*}$. Then $U \in \mathcal{F}_{\min }$ and $\jmath\left(\mathcal{F}_{\min }\right) \geqslant \mathcal{V}_{q_{\mathcal{D}}}([\mathcal{F}])$, the $q_{\mathcal{D}}$-neighbourhood filter at $[\mathcal{F}]$. Thus $\mathcal{F}_{\min } \geqslant \mathfrak{J}^{-1}\left(\mathcal{V}_{q_{\mathcal{D}}}([\mathcal{F}])\right)$, and since the latter filter is in $\mathcal{C}$, it follows from the minimality of $\mathcal{F}_{\min }$ that $\mathcal{F}_{\min }=\mathcal{J}^{-1}\left(\mathcal{V}_{q_{\mathcal{D}}}([\mathcal{F}])\right)$. Since $U \in \mathcal{F}_{\min }$, there is a $q_{\mathcal{D}}$-open set $W \in \mathcal{V}_{q_{\mathcal{D}}}([\mathcal{F}])$ such that $j^{-1}(W) \subseteq U$. To see that $W \subseteq U^{*}$, let $[\mathcal{G}] \in W$; then $\jmath\left(\mathcal{G}_{\min }\right) \stackrel{q_{\mathcal{D}}}{\rightarrow}[\mathcal{G}]$, and since $W$ is $q_{\mathcal{D}}$-open, $\jmath^{-1}(W) \in \mathcal{G}_{\text {min }}$, which implies $U \in \mathcal{G}_{\min }$, and hence $[\mathcal{G}] \in U^{*}$. Thus $U^{*}$ is $q_{\mathcal{D}}$-open.

The preceding theorem shows that $\left(\left(X^{*}, \mathcal{C}^{*}\right), \jmath\right.$ ) is (up to equivalence) the coarsest $T_{2}$, diagonal completion of a $T_{2}$, diagonal Cauchy space $(X, \mathcal{C})$; for this reason, $\left(\left(X^{*}, \mathcal{C}^{*}\right), \jmath\right)$ will be called the coarse diagonal completion of $(X, \mathcal{C})$. The next example shows that a $T_{2}$, diagonal Cauchy space may have $T_{2}$, diagonal completions which are not equivalent to the coarse diagonal completion. 
EXAMPLE 2.4. Let $X$ be the set $Q$ of rational numbers, and let $\mathcal{C}$ be the usual Cauchy structure for $Q$. In this case, we can make the usual identification between the set $Q^{*}$ and the set $R$ of real numbers, in which case $\jmath: Q \rightarrow R$ becomes the identity injection. The Cauchy structure $\mathcal{C}^{*}$ on $R$ of the coarse diagonal completion is the usual (complete) Cauchy structure for $R$ which gives rise to the usual topology. Next, consider the finest topology $\tau$ on $R$ which contains all sets open in the usual topology along with the set $Q$. If $y \in R \backslash Q, \mathcal{V}_{\tau}(y)$ has a filter base of open intervals of the form $(y-\varepsilon, y+\varepsilon)$, where $\varepsilon>0$, but if $y \in Q, \mathcal{V}_{\tau}(y)$ has a filter base of sets of the form $(y-\varepsilon, y+\varepsilon) \cap Q$. If $\mathcal{C}^{\prime}=\left\{\mathcal{F} \in \mathrm{F}(R):\right.$ there is $y \in R$ such that $\left.\mathcal{F} \geqslant \mathcal{V}_{\tau}(y)\right\}$, then $\left(\left(R, \mathcal{C}^{\prime}\right), \jmath\right)$ is a $T_{2}$, diagonal completion of $(X, \mathcal{C})$ which is obviously not equivalent to $\left(\left(R, \mathcal{C}^{*}\right), \jmath\right)$.

Recall that a Cauchy space $(X, \mathcal{C})$ is $T_{3}$ if it is $T_{2}$ and has the property that $c l_{q_{\mathcal{C}}}(\mathcal{F}) \in \mathcal{C}$ wherever $\mathcal{F} \in \mathcal{C}$. The next result shows that the coarse diagonal completion is at least as fine as any $T_{3}$ completion of a $T_{2}$, diagonal Cauchy space.

Proposition 2.5. If $\left(\left(X^{*}, \mathcal{D}\right), \jmath\right)$ is any $T_{3}$ completion (in standard form) of a $T_{2}$, diagonal Cauchy space $(X, \mathcal{C})$, then $\mathcal{C}^{*} \subseteq \mathcal{D}$.

Proof: If $\mathcal{A} \in \mathcal{C}^{*}$, then $\mathcal{A} \geqslant \mathcal{F}_{\min }^{*}$, for some $\mathcal{F} \in \mathcal{C}$. But $\mathcal{F}_{\min }^{*} \geqslant c l_{\mathcal{D}} \jmath\left(\mathcal{F}_{\min }\right)$, and it follows by the $T_{3}$ property that $\mathcal{A} \in \mathcal{D}$.

The next theorem establishes that the coarse diagonal completion preserves uniformisability.

THEOREM 2.6. Let $(X, \mathcal{U})$ be a $T_{2}$ uniform space, and let $\mathcal{C}=\{\mathcal{F} \in \mathbf{F}(X)$ : $\mathcal{U} \leqslant \mathcal{F} \times \mathcal{F}\}$ be the associated Cauchy structure. Let $\left(\left(X^{*}, \mathcal{U}^{\wedge}\right), \jmath\right)$ be the uniform completion of $(X, \mathcal{U})$ in standard form, and let $\mathcal{C}^{\wedge}=\left\{\mathcal{A} \in \mathbf{F}\left(X^{*}\right): \mathcal{U}^{\wedge} \leqslant \mathcal{A} \times \mathcal{A}\right\}$. Then $C^{*}=C^{\wedge}$.

Proof: Observe that $(X, \mathcal{C})$ is a $T_{2}$, diagonal Cauchy space, and $\left(\left(X^{*}, \mathcal{C}^{\wedge}\right), \jmath\right)$ is the "uniformisable Cauchy completion" of $(X, \mathcal{C})$. The latter completion is clearly a $T_{2}$, diagonal completion, so $\mathcal{C}^{\wedge} \subseteq \mathcal{C}^{*}$ follows by Theorem 2.3. But any uniformisable completion is also $T_{3}$, so it follows by Proposition 2.5 that $\mathcal{C}^{*} \subseteq \mathcal{C}^{\wedge}$.

Indeed, the following more general result follows as in the preceding proof from Theorem 2.3 and Proposition 2.5.

Corollary 2.7. Any $T_{3}$, diagonal completion of a $T_{3}$, diagonal Cauchy space is the coarse diagonal completion.

The next theorem gives a condition which is necessary and sufficient for the coarse diagonal completion of a $T_{3}$, diagonal Cauchy space to be $T_{3}$.

Let $(X, \mathcal{C})$ be a $T_{2}$, diagonal Cauchy space, and for $A \subseteq X$, let $A^{* *}=\left\{[\mathcal{F}] \in X^{*}\right.$ : $\mathcal{F}_{\min }$ has a trace on $\left.A\right\}$. Note that $A^{*} \subseteq A^{* *}$ and $\jmath^{-1}\left(A^{* *}\right)=c l_{q_{\mathcal{C}}} A$ for all $A \subseteq X$; furthermore, if $\mathcal{F} \in \mathbf{F}(X)$, then the filter $\mathcal{F}^{* *}$ generated by $\left\{F^{* *}: F \in \mathcal{F}\right\}$ is a proper 
filter on $X^{*}$.

ThEOREM 2.8. Let $(X, \mathcal{C})$ be a $T_{3}$, diagonal Cauchy space. Then the coarse diagonal completion of $(X, \mathcal{C})$ is $T_{3}$ if and only if $\mathcal{F}_{\min }^{*}=\mathcal{F}_{\min }^{* *}$, for each $\mathcal{F} \in \mathcal{C}$.

ProOF: Let $q^{*}$ be the convergence structure associated with $\left(X^{*}, \mathcal{C}^{*}\right)$. One easily verifies that for any $\mathcal{F} \in \mathcal{C}, \mathcal{F}_{\text {min }}^{* *}=c l_{q^{*}}\left(\mathcal{F}_{\text {min }}\right)$. If $\left(X^{*}, \mathcal{C}^{*}\right)$ is $T_{3}$, then $\mathcal{F}_{\text {min }}^{*}=\mathcal{F}_{\text {min }}^{* *}$ follows immediately. On the other hand, if $\mathcal{F}_{\min }^{*}=\mathcal{F}_{\min }^{* *}$ for every $\mathcal{F} \in \mathcal{C}$, then $\mathcal{F}_{\min }^{*}$ has a filter base of $q^{*}$-closed sets for every $\mathcal{F} \in \mathcal{C}$, and therefore $\left(X^{*}, \mathcal{C}^{*}\right)$ is $T_{3}$.

The following example shows that the coarse diagonal completion need not preserve either the $T_{3}$ property or total boundedness.

EXAMPLE 2.9. Let $X$ be an infinite set, partitioned into infinite subsets $\left\{X_{i}: i \in N\right\}$, where $N$ is the set of natural numbers. For each $i \in N$, let $\mathcal{G}_{i}$ be the filter on $X$ with filter base $\left\{X_{i} \backslash A: A\right.$ a finite subset of $\left.X_{i}\right\}$. Let $\mathcal{C}=\{\mathcal{H} \in \mathbf{F}(X): \exists i \in N$ such that $\left.\mathcal{H} \geqslant \mathcal{G}_{i}\right\} \cup\{\mathcal{F}: \mathcal{F}$ a free ultrafilter on $X\} \cup\{\dot{\boldsymbol{x}}: \boldsymbol{x} \in X\}$. Note that $\mathcal{C}$ is a Cauchy structure on $X$ and $q_{\mathcal{C}}$ is discrete; hence $(X, \mathcal{C})$ is a totally bounded, $T_{3}$, diagonal Cauchy space.

Let $\mathcal{G}$ be any free ultrafilter on $X$ such that each $G \in \mathcal{G}$ has an infinite intersection with infinitely many of the $X_{i}$ 's. Then $\mathcal{F}=\mathcal{G}_{\min }$ and $\mathcal{G}_{\min }^{*}=\jmath(\mathcal{G}) \cap[\dot{\mathcal{G}}]$, but $\mathcal{G}_{\min }^{* *}$ has the property that for each $G \in \mathcal{G}, G^{* *}$ contains infinitely many of the $\left[\mathcal{G}_{i}\right]$ 's. Thus $\mathcal{G}_{\min }^{* *} \neq \mathcal{G}_{\min }^{*}$, so by Theorem $2.8,\left(X^{*}, \mathcal{C}^{*}\right)$ is not $T_{3}$. Furthermore, $\left(X^{*}, \mathcal{C}^{*}\right)$ is not totally bounded; otherwise, $\left(\left(X^{*}, q^{*}\right), \jmath\right)$ would be a $T_{2}$, topological compactification of $\left(X, q_{c}\right)$, which would imply that $\left(X^{*}, \mathcal{C}^{*}\right)$ is $T_{3}$.

It comes as no surprise that the coarse diagonal completion for $T_{2}$, diagonal Cauchy spaces does not behave "functorially". For if $(Q, \mathcal{C})$ and $\left(R, \mathcal{C}^{\prime}\right)$ are the $T_{2}$, diagonal Cauchy spaces defined in Example 2.4 and $\jmath:(Q, \mathcal{C}) \rightarrow\left(R, \mathcal{C}^{\prime}\right)$ is the identity injection, then $J$ is Cauchy continuous, but $J$ obviously has no Cauchy continuous extension $\jmath^{*}:\left(Q^{*}, \mathcal{C}^{*}\right) \rightarrow\left(R^{*},\left(\mathcal{C}^{\prime}\right)^{*}\right)=\left(R, \mathcal{C}^{\prime}\right)$

There is, however, a completion functor on the category $T_{2} D C H Y$ of $T_{2}$ diagonal Cauchy spaces and Cauchy continuous maps. This completion is, for obvious reason, called the fine diagonal completion and is denoted (in standard form) by $\left(\left(X^{*}, \mathcal{C}^{\star}\right), \jmath\right)$. The construction of this completion is briefly described below.

Given an arbitrary $T_{2}$ Cauchy space $(X, \mathcal{C})$, let $\left(\left(X^{*}, \mathcal{W}\right), \jmath\right)$ denote the Wyler completion (see [5]) of $(X, \mathcal{C})$, where $\mathcal{W}=\left\{\mathcal{A} \in \mathbf{F}\left(X^{*}\right)\right.$ : there is $\mathcal{F} \in \mathcal{C}$ such that $\mathcal{A} \geqslant \jmath(\mathcal{F}) \cap[\dot{\mathcal{F}}]\}$. The Wyler completion defines a completion functor on the category $\mathrm{T}_{2} \mathrm{CHY}$ of all $\mathrm{T}_{2}$ Cauchy spaces, but this completion does not preserve the diagonal property. Let $\mathcal{C}^{\star}$ be the complete Cauchy structure on $X^{*}$ associated with the topological modification $\tau_{q_{\mathcal{W}}}$ of $q_{\mathfrak{W}}$. 
Now let $(X, \mathcal{C})$ be a $T_{2}$, diagonal Cauchy space. It is clear that $\mathcal{C}^{*} \subseteq \mathcal{C}^{\star} \subseteq \mathcal{W}$. Since $\jmath:(X, \mathcal{C}) \rightarrow\left(X^{*}, \mathcal{C}^{*}\right)$ and $\jmath:(X, \mathcal{C}) \rightarrow\left(X^{*}, \mathcal{W}\right)$ are both Cauchy embeddings, $\jmath:$ $(X, \mathcal{C}) \rightarrow\left(X^{*}, \mathcal{C}^{\star}\right)$ is also a Cauchy embedding, and hence $\left(\left(X^{*}, \mathcal{C}^{\star}\right), \jmath\right)$ is a $T_{2}$, diagonal completion of $(X, \mathcal{C})$. Since $\mathcal{W}$ is the finest $T_{2}$ completion structure for $(X, \mathcal{C})$ in standard form, $\mathcal{C}^{\star}$ is the finest diagonal completion structure for a $T_{2}$, diagonal Cauchy space $(X, \mathcal{C})$ in standard form. Furthermore, since the Wyler completion and the topological modification are both functorial, we obtain the following extension theorem for the fine diagonal completion.

Theorem 2.10. Let $(X, \mathcal{C})$ and $(Y, \mathcal{D})$ be $T_{2}$, diagonal Cauchy spaces, and let $f:(X, \mathcal{C}) \rightarrow(Y, \mathcal{D})$ be Cauchy continuous. Then there is a unique Cauchy continuous function $f^{\star}:\left(X^{*}, \mathcal{C}^{\star}\right) \rightarrow\left(Y^{*}, \mathcal{D}^{\star}\right)$ such that the following diagram commutes:

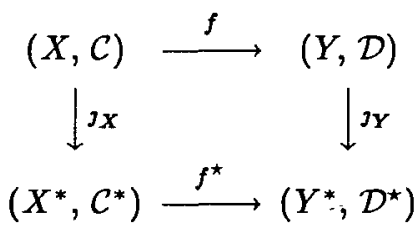

As noted in the remarks following Example 2.9, Theorem 2.10 is not valid for the coarse diagonal completion. However, the latter completion does exhibit an interesting extension property relative to a weaker type of continuity.

Let $(X, q)$ and $(Y, p)$ be topological spaces. $f:(X, q) \rightarrow(Y, p)$ is said to be $\boldsymbol{\theta}$-continuous if, for every $x \in X$ and every neighbourhood $V$ of $f(x)$, there is a neighbourhood $U$ of $x$ such that $f\left(c l_{q} U\right) \subseteq c l_{p}(V)$. If $(X, \mathcal{C})$ and $(Y, \mathcal{D})$ are complete Cauchy spaces such that $q_{\mathcal{c}}$ and $q_{\mathcal{D}}$ are topologies, we define $f:(X, \mathcal{C}) \rightarrow(Y, \mathcal{D})$ to be $\theta$-continuous if $f:\left(X, q_{\mathcal{C}}\right) \rightarrow\left(Y, q_{\mathcal{D}}\right)$ is $\theta$-continuous.

Theorem 2.11. Let $(X, \mathcal{C})$ and $(Y, \mathcal{D})$ be $T_{2}$, diagonal Cauchy spaces and let $f:(X, \mathcal{C}) \rightarrow(Y, \mathcal{D})$ be Cauchy continuous. Then there is a $\theta$-continuous function $f^{*}:\left(X^{*}, \mathcal{C}^{*}\right) \rightarrow\left(Y^{*}, \mathcal{D}^{*}\right)$ such that the following diagram commutes:

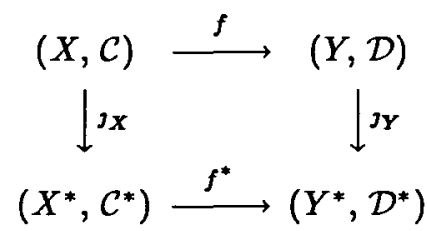

ProOF: Let $q^{*}$ be the topology on $X^{*}$ induced by $\mathcal{C}^{*}$ and $p^{*}$ the topology on $Y^{*}$ induced by $\mathcal{D}^{*}$. If $[\mathcal{F}]_{\mathcal{c}} \in X^{*}$, let $f^{*}\left([\mathcal{F}]_{\mathcal{C}}\right)=[f(\mathcal{F})]_{\mathcal{D}}$. In particular, if $\mathcal{F}=\dot{x}$, then $f^{*}\left([\dot{x}]_{\mathcal{C}}\right)=[f(\dot{x})]_{\mathcal{D}}$, and it follows that the above diagram commutes.

Next, we show that for any $A \subseteq X, f^{*}\left(c l_{q^{*}}\left(A^{*}\right)\right) \subseteq c l_{p^{*} \jmath_{Y}}(f(A))$. Given $A \subseteq X$, let $[\mathcal{F}] \in c l_{q^{*}}\left(A^{*}\right)$. Since $F^{*} \cap A^{*} \neq \emptyset$ for all $F \in \mathcal{F}_{\min }, F \cap A \neq \emptyset$ for all $F \in \mathcal{F}_{\min }$, 
and therefore $f\left(\mathcal{F}_{\min }\right)$ has a trace on $f(A)$. Consequently, $(f(\mathcal{F}))_{\min }^{*}$ has a trace on $\jmath_{Y} f(A)$, which implies that $f^{*}\left([\mathcal{F}]_{\mathcal{C}}\right)=[f(\mathcal{F})]_{\mathcal{D}} \in c l_{p^{*}} \jmath_{Y}(f(A))$.

Finally, let $[\mathcal{F}]_{c} \in X^{*}$ and $V^{*} \in(f(\mathcal{F}))_{\min }^{*}$, where $V \in(f(\mathcal{F}))_{\min }$. Choose $U \in \mathcal{F}_{\min }$ such that $f(U) \subseteq V$. Then $U^{*}$ is a $q^{*}$-neighbourhood of $[\mathcal{F}]_{\mathcal{C}}$ such that $f^{*}\left(c l_{q^{*}}\left(U^{*}\right)\right) \subseteq c l_{p^{*}} \jmath_{Y}(f(U)) \subseteq c l_{p^{*}}\left(V^{*}\right)$.

\section{REFERENCES}

[1] C.H. Cook and H.R. Fischer, 'Regular convergence spaces', Math. Ann. 174 (1967), 1-7.

[2] H.J. Kowalsky, 'Limesräume und Komplettierung', Math. Nachr. 12 (1954), 301-340.

[3] E.E. Reed, 'Completions of uniform convergence spaces', Math. Ann. 194 (1971), 83-108.

[4] G.D. Richardson and D.C. Kent, 'Probabilistic convergence spaces', J Austral. Math. Soc. (to appear).

[5] O. Wyler, 'Ein Komplettierungfunktor für Uniforme Limesräume', Math. Nachr. 46 (1970), 1-12.

Department of Pure and Applied Mathematics Washington State University

Pullman WA 99164-3113

United States of America
Department of Mathematics University of Central Florida Orlando FL 32816

United States of America 